\title{
Screening of fungal strains of the genus Beauveria for their ability to form endophytic relations with Phaseolus plants
}

\author{
Natalia Volkova ${ }^{1}$, Maksim Levchenko ${ }^{I}$, Alibek Uspanov², and Georgiy Lednev ${ }^{1 *}$ \\ ${ }^{1}$ All-Russian Institute of Plant Protection, Podbelskogo 3, 196608, St. Petersburg, Pushkin, Russia \\ ${ }^{2}$ Kazakh scientific research institute of plant protection and quarantine, Almaty, Republic of \\ Kazakhstan
}

\begin{abstract}
Screening of 11 strains of Beauveria fungi based on their colonization rate (endophytic activity) on bean plants showed that all the cultures tested belonging either to Beauveria bassiana or Beauveria pseudobassiana are able to colonize the plants. Significant differences were revealed between the strains in their endophytic activity. Three strains of fungi with the highest endophytic activity were found and a slight growthstimulating effect was revealed for the four strains. The relative increase in plant height was significantly higher compared to both control and other fungal cultures.
\end{abstract}

\section{Introduction}

Endophytes are microorganisms that constantly live or pass part of their life cycle in healthy plant tissues without causing pathological changes [1]. As a rule, these are bacteria and fungi that either display no effects or mutually beneficial relationships with their host plants.

To date, there is a significant number of reports of the detection of endophytic fungi in both cultivated and wild plants [2]. In addition, there is a large number of publications describing successful colonization of plants by entomopathogenic fungi. In particular, Beauveria bassiana was successfully injected into corn, cotton, tomatoes, beans, and many other plants [3].

The use of entomopathogenic fungi as endophytes indicate their suitability not only as mycoinsecticides but as multifunctional bioformulations as well $[4,5]$.

\footnotetext{
*Corresponding author: georgijled@mail.ru
} 


\section{Materials and Methods}

In the experiments, 11 strains of their fungi of the genus Beauveria from the collection of the All-Russian Institute of Plant Protection were used. As plants for inoculation, common bean Phaseolus vulgaris, cultivar Lyudmila was used. The substrate for growing plants was a sterile soil-sand mixture in a ratio of 1:5. Prior to sowing, the seeds were surface-sterilized. The plants were treated a week after emergence (phase of the third true leaf) by watering with a conidial suspension, $10 \mathrm{ml}$ for each plant, at the concentration of $10^{8}$ conidia $/ \mathrm{mL}$.

After one and two weeks, 3 parts of the plants (leaf, stem and root) were selected for testing for the level of their colonization by fungi. Previously, basic biometric indicators (stem and root length) were estimated for the assayed plants. The samples were washed and individually sterilized with $1 \%$ sodium hypochlorite for 2 min and $70 \%$ ethyl alcohol for 2 min, followed by triple washing with distilled water $[5,7]$. Then the samples were cut into small parts and placed in Petri dishes on solid Chapek's medium with addition of lactic acid $(2 \mathrm{ml} / \mathrm{L})$. To control the quality of surface sterilization, the plant tissue samples were pressed against the medium's surface and removed. Then the cups were examined every day, starting from the 4 th day, to observe the growth of fungal colonies.

\section{Results and Discussion}

It was found that all tested cultures are able to colonize the plants. Significant differences between the strains by their endophytic activity were revealed. The highest ability to inhabit plants $(100 \%$ colonization of plant samples two weeks after fungal contamination of the plants) has been demonstrated by three cultures referred to as BBK-1, BCu3-B-08 and BBKZh-06 (Fig. 1A). They have also shown the highest intensity of colonization was also noted (28-31\% of fragments showing the fungal presence).

For the four strains of BBK-1, BCu3-B-08, BBKZh-06, and BLDB-09, the systemic distribution of the fungus over the plant was found (the fungi were localized in the roots, stalk, and leaves of the plants) (Fig. 1B). No correlation between of the species allocation of the strains and their endophytic activity was revealed. For all studied cultures, high level of colonization was observed in plant stems. Among the three cultures that showed the highest level of plant colonization, two belong to B. bassiana and one to Beauveria pseudobassiana.

For the four strains BCu22-07, BLDB-09, BLDR-09 and BCu1-D-09, a slight growth-promoting effect was revealed (Fig. 2). The relative increase of the treated plant height was significantly higher as compared to both control and other fungal cultures. 


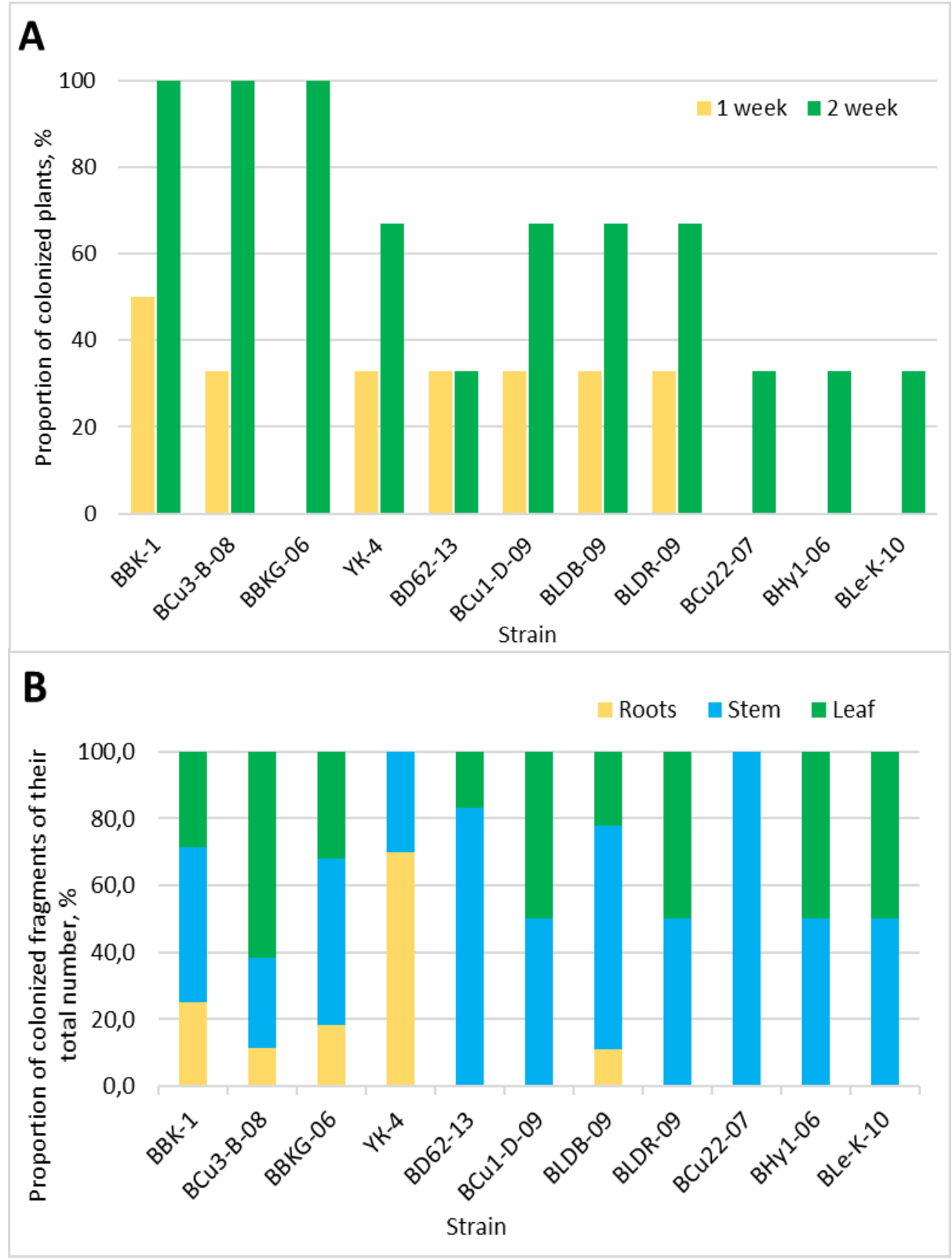

Fig. 1. Colonization of bean plants by strains of fungi of the genus Beauveria. A. Proportion of colonized plants B. Proportion of colonized fragments of their total number (for the second week) 


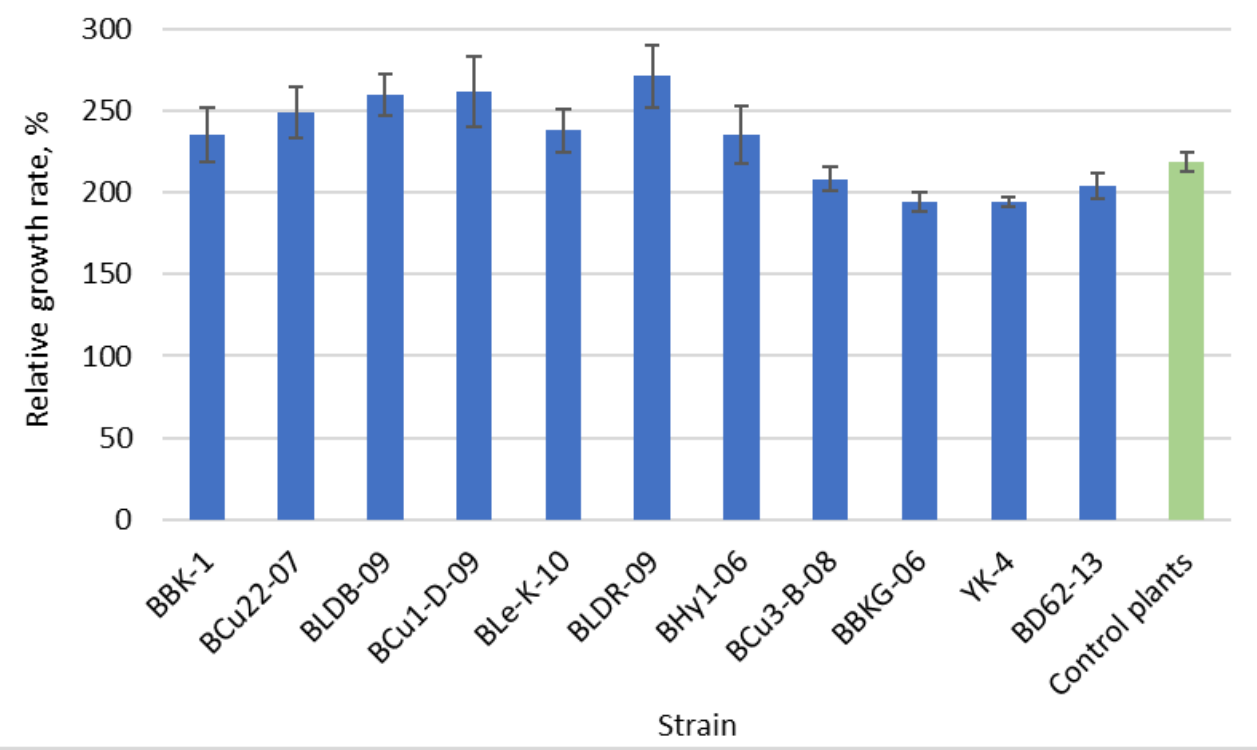

Fig. 2. Growth stimulating effect in inoculation of bean plants with strains of the genus Beauveria (for the second week)

\section{Conclusion}

Both B. bassiana and B. pseudobassiana were found to have plant growth stimulating effect and this ability was dependent upon the strain. These observations are in agreement with those obtained in other fungus-plant systems and support the idea of development of multifunctional formulations on the basis of enthomopathogenic fungi $[4,5]$.

The research is supported by Ministry of Education and Science of Kazakhstan Republic, grant \# AP 05135810.

\section{References}

1. Vega F.E. J. Invertebr. Pathol. 98(3), 277-279 (2008)

2. Vega F.E. Mycologia. 110, 4-30 (2018)

3. Schulz, B. and Boyle, C., Mycol. Res. 109, 661-686 (2005)

4. Ownley B.H., K.D. Gwinn, and F.E. Vega, BioControl. 55(1), 113-128 (2010)

5. Muvea, A. M., R. Meyhofer, S. Sevgan, H. Poehling, S. Ekesi, and N. K. Maniania. PLoS One 9, e108242 (2014) 